\title{
Bioinformatic analysis of microRNA-mRNA expression profiles of bladder tissue induced by bladder outlet obstruction in a rat model
}

\author{
LIU JIAN DUAN*, QI FENG CAO*, DING XU, HAI LONG LIU and JUN QI \\ Department of Urology, Xin Hua Hospital Affiliated to Shanghai Jiao Tong University School of Medicine, \\ Shanghai 200092, P.R. China
}

Received March 4, 2016; Accepted February 23, 2017

DOI: $10.3892 / \mathrm{mmr} .2017 .7188$

\begin{abstract}
Various microRNAs (miRNAs) have previously been demonstrated to exhibit an association with the process of bladder remodeling, induced by bladder outlet obstruction (BOO). However, little is known about miRNA and gene expression profiles and the molecular mechanism underlying bladder pathophysiological alterations. The present study used bioinformatic analysis technology to examine the altered miRNA and mRNA expression profiles of bladder tissue in a rat model of $\mathrm{BOO}$ and validate the involved signaling pathways. The gene expression profile data was downloaded from Gene Expression Omnibus (GEO), and the differentially expressed genes (DEGs) and differentially expressed miRNAs (DEMs) were screened. Potential target genes of DEMs were predicted. The target genes and DEGs were used for further gene ontology (GO) analysis followed by Kyoto Encyclopedia of Genes and Genomes (KEGG) pathway analysis using the Database for Annotation, Visualization and Integrated Discovery. The present study additionally constructed a DEM-DEG interaction network. A total of 9 DEMs (3 upregulated and 6 downregulated) were identified; 664 DEGs were screened. KEGG analysis revealed that the DEGs were involved in the regulation of the actin cytoskeleton, extracellular matrix (ECM) remodeling, cell adhesion and the cell cycle. Additionally, KEGG classification indicated that these genes were important in angiogenesis, and in the $\mathrm{p} 53$ and transforming growth factor- $\beta$ signaling pathways. Notably, rno-miRNA (miR)-26b and rno-miR-101b were the two larger nodes of the 7 obstruction-associated
\end{abstract}

Correspondence to: Professor Jun Qi, Department of Urology, Xin Hua Hospital Affiliated to Shanghai Jiao Tong University School of Medicine, 1665 Kong Jiang Road, Shanghai 200092, P.R. China E-mail: jasonqi@sh163.net

*Contributed equally

Key words: bladder outlet obstruction, extracellular matrix, microRNA, bioinformatic analysis, gene expression profiles
DEMs and interacted with 32 and 27 DEGs, respectively. On removal of obstruction, few DEMs were present; however, 370 genes exhibited the opposite expression trend. The majority of pathways enriched for the DEGs were identified and were associated with ECM-receptor interaction and focal adhesion. In the DEM-DEG regulatory network, miR-495, miR-494 and their target genes were significantly differentially expressed. The present study demonstrated that miRNAs and genes may be potential biomarkers of bladder remodeling induced by $\mathrm{BOO}$, and additionally provided novel insights into the molecular mechanisms underlying this disorder.

\section{Introduction}

Benign prostate hyperplasia accompanied by bladder outlet obstruction (BOO) has been revealed to affect $30 \%$ of men $>60$ years old (1). Urinary bladder dysfunction is associated with bladder pathophysiological alterations resulting from BOO. The pathogenesis of $\mathrm{BOO}$ has become a primary concern and has been widely investigated. Experimental and clinical studies have previously demonstrated that the symptomatic presentation of BOO may be associated with a physiopathological cascade accompanied by a variety of morphologically and functionally degenerative alterations within the urinary bladder wall $(2,3)$. Opinion is spreading that, prior to acting as a direct initiator in the urinary symptoms, the bladder is the organ first affected by this disease (4). Further studies are required to fully elucidate the underlying pathogenesis of bladder remodeling resulting from $\mathrm{BOO}$.

MicroRNAs (miRNAs) are non-coding RNA molecules, $\sim 22$ nucleotides in length, that regulate protein expression post-transcriptionally by targeting mRNAs for cleavage or translational repression. miRNAs bind to complementary sequences in the 3'-untranslated region of target mRNAs $(5,6)$. Each miRNA is predicted to regulate numerous mRNAs. It has previously been demonstrated that miRNAs are fundamental in numerous biological processes, and may be termed multifunctional mediators (6,7). Previous studies have additionally demonstrated that miRNAs are associated with cardiac and muscle remodeling $(8,9)$, and hepatofibrogenesis (10). Alterations in the expression of miRNAs appears to 
be important for tissue remodeling, suggesting their potential as biomarkers and therapeutic targets for this process (11). miRNA profiling and bioinformatic tools has resulted in the identification of key miRNAs and target genes involved in bladder cancer (12). Regarding obstructed bladder remodeling, miRNA (miR)-29 repression is involved in extracellular matrix (ECM) remodeling and altered stiffness (13), and detrusor induction of miR-132/122 is associated with cell growth and gene expression (14). Our previous study demonstrated that miR-133 modulates the transforming growth factor (TGF)- $\beta 1$-induced bladder smooth muscle cell hypertrophic and fibrotic response by targeting connective tissue growth factor (15). Despite the known association of various miRNAs with bladder remodeling, little is known regarding the global expression alterations of miRNAs and mRNAs in the development and progression of bladder remodeling associated with bladder dysfunction.

The present study aimed to investigate miRNA and mRNA expression profiles of bladder tissue following BOO, in a rat model. Additionally, the present study aimed to present a novel and comprehensive molecular signature to contribute to and support future studies on the physiopathological cascade associated with this condition. An interaction network of miRNA-mRNA was constructed and the potential BOO-associated underlying mechanisms were investigated. The data provided novel and robust information regarding the underlying pathogenesis of bladder remodeling.

\section{Materials and methods}

Affymetrix microarray data. The microarray dataset GSE47080, deposited by Ekman et al (13), was downloaded from the Gene Expression Omnibus (GEO; www.ncbi.nlm. nih.gov/geo/) database. This data was obtained from rats in which the urethra was partially ligated and the urinary bladder removed 10 days ( $n=6$; obs10d group) or 6 weeks $(n=8$; obs6w group) following obstruction. Sham-operated rats $(n=6)$ served as a control. An additional group of rats ( $n=6$; deobs10d group) underwent a further operation 6 weeks following surgery and the obstruction was removed. The urinary bladder was obtained from these rats 10 days following the obstruction removal. A total of 26 bladder specimens (including the urothelium) were therefore used for the development of mRNA and miRNA expression profiles, based on the GPL6247 Affymetrix Rat Gene 1.0 ST Array and GPL16384 Affymetrix Multispecies miRNA-3 Array platforms, respectively (Affymetrix, Inc., Santa Clara, CA, USA).

Data preprocessing. The raw data of gene and miRNA expression profiles was preprocessed using R/Bioconductor affy (bioconductor.org/packages/release/bioc/html/affy. html) (16). The expression values were measured and normalization was performed using the robust multiarray average algorithm (17).

Analysis of differentially expressed genes (DEGs) and differentially expressed miRNAs (DEMs). DEGs and DEMs in obs10d vs. sham control, obs6w vs. sham control and obs6w vs. deobs10d groups were identified using the R/Bioconductor package linear models for microarray data (www.bioconductor.
Table I. KEGG pathways enriched by obstruction-associated differentially expressed genes.

\begin{tabular}{lc} 
Term & P-value \\
\hline no04510: Focal adhesion & $1.84 \mathrm{E}-06$ \\
rno04512: ECM-receptor interaction & $5.83 \mathrm{E}-06$ \\
rno05412: Arrhythmogenic right ventricular & $6.48 \mathrm{E}-05$ \\
cardiomyopathy & \\
rno05414: Dilated cardiomyopathy & $3.87 \mathrm{E}-04$ \\
rno04270: Vascular smooth muscle contraction & $7.78 \mathrm{E}-04$ \\
rno05410: Hypertrophic cardiomyopathy & $8.77 \mathrm{E}-04$ \\
rno04810: Regulation of actin cytoskeleton & 0.001504668 \\
rno04020: Calcium signaling pathway & 0.002489785 \\
rno05222: Small cell lung cancer & 0.003042027 \\
rno05200: Pathways in cancer & 0.005441483 \\
rno00564: Glycerophospholipid metabolism & 0.006966525 \\
rno04115: p53 signaling pathway & 0.009762513 \\
rno04080: Neuroactive ligand-receptor & 0.014532004 \\
interaction & \\
rno04110: Cell cycle & 0.016275599 \\
rno04010: MAPK signaling pathway & 0.016666232 \\
rno04960: Aldosterone-regulated sodium & \\
reabsorption & 0.019224962 \\
rno00565: Ether lipid metabolism & 0.033933454 \\
rno04350: TGF- $\beta$ signaling pathway & 0.036718279 \\
rno05020: Prion diseases & 0.037254423 \\
rno05211: Renal cell carcinoma & 0.039107910 \\
rno00590: Arachidonic acid metabolism & 0.041527221 \\
rno04360: Axon guidance & 0.041739230 \\
rno04514: Cell adhesion molecules & 0.043318929 \\
& \\
&
\end{tabular}

KEGG, Kyoto Encyclopedia of Genes and Genomes; MAPK, mitogen activated protein kinase; ECM, extracellular matrix.

org/packages/release/bioc/html/limma.html) (18). The threshold for screening DEGs and DEMs was a log fold change $\geq 0.5$. To reduce the false positive rate, the P-value was adjusted as adj.p.val<0.05 by the Benjamini and Hochberg method (19) using the Bioconductor's multtest package. In addition, the DEGs and DEMs in obs10d vs. sham control and obs6w vs. sham control groups, and obs6w vs. sham control and obs6w vs. deobs10d groups were compared using the VENNY version 2.1 (bioinfogp.cnb.csic.es/tools/venny/). The hierarchical clustering heat map of the DEGs and DEMs was generated and visualized using $\mathrm{R}$ package gplots (20).

Functional enrichment analysis. The Kyoto Encyclopedia of Genes and Genomes (KEGG; www.genome.ad.jp/kegg/) (21) was used to integrate and interpret the significant pathway information of large-scale molecular data sets. The Database for Annotation, Visualization and Integrated Discovery (DAVID; david.ncifcrf.gov/) (22) was used to annotate and visualize the integrated pathway information. The present study performed KEGG pathway analysis for DEGs with the same expression trend in obs10d vs. sham control and obs6w vs. sham control groups, and DEGs with inverse expression 
A

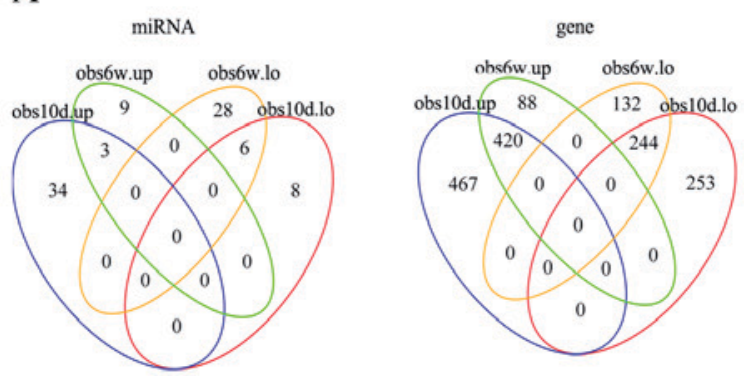

$\mathrm{C}$

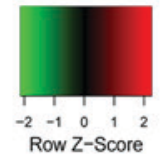

B miRNA

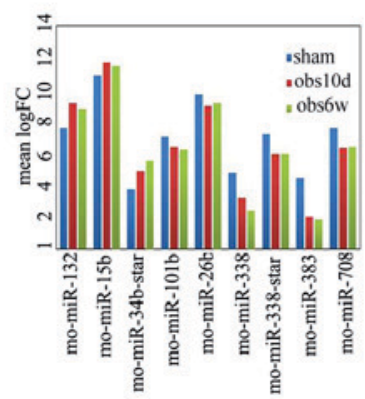

D

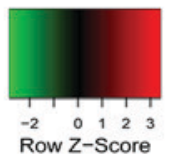

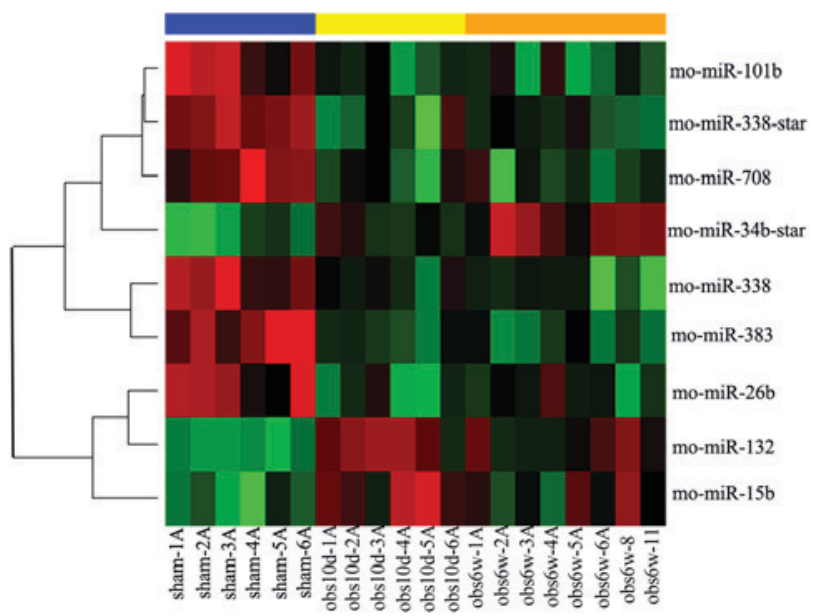

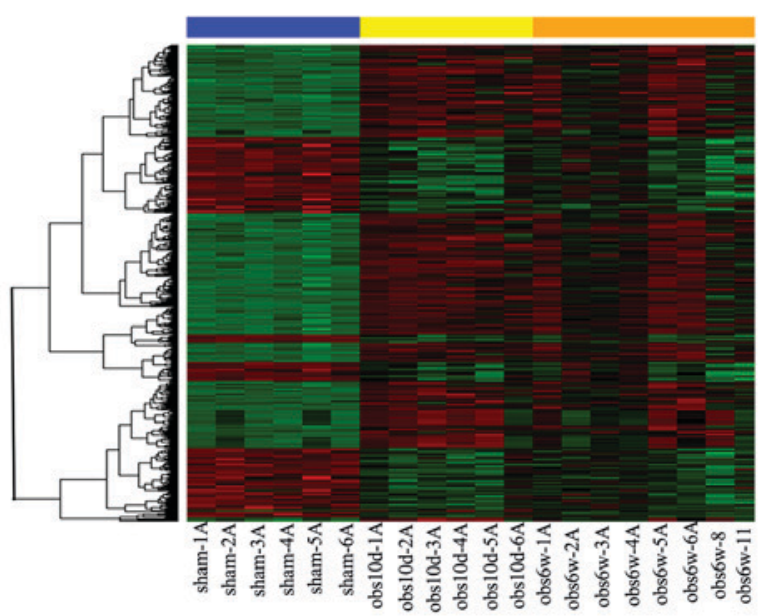

Figure 1. DEMs and DEGs induced by bladder outlet obstruction. (A) Venn plots of DEMs and DEGs. (B) Expression profiles of 9 obstruction-associated DEMs. Heat map plots of (C) DEMs and (D) DEGs. Columns represent rat bladder samples, from the sham10d (blue; $\mathrm{n}=6$ ), obs10d (yellow; $\mathrm{n}=6$ ) and obs6w (orange; $n=8$ ) groups. The colors depict high (red) and low (green) level of miRNA or gene expression. The color alteration from green to black to red represents increasing expression. Right vertical axis represents clustering. DEGs, differentially expressed genes; DEMs, differentially expressed microRNAs; obs10d, urinary bladder removed 10 days following obstruction; obs6w, urinary bladder removed 6 weeks following obstruction; miRNA, microRNA.

trend in obs6w vs. sham control and obs6w vs. deobs10d groups, using the DAVID online tool. The significant KEGG pathways were enriched with $\mathrm{P}<0.05$.

Construction of miRNA-gene interaction network. To examine the interaction between DEMs and DEGs, the present study used miRanda (www.microrna.org/microrna/home) to predict the target genes of miRNAs. The comparison of DEGs and target genes resulted in the construction of the miRNA-gene interaction network.

\section{Results}

Data preprocessing and identification of DEMs and DEGs. Following preprocessing, data was successfully normalized. A boxplot of preprocessed miRNA and gene profiles was constructed (data not shown). Compared with the sham control group, the present study identified 51 DEMs (37 up and 14 downregulated) and 1,384 DEGs (887 up and 497 downregulated) in the obs10d group, and 46 DEMs (12 up and 34 downregulated) and 884 DEGs (508 up and 376 downregulated) in the obs6w group. Furthermore, 27 DEMs (21 up and 6 downregulated) and 740 DEGs (490 up and 250 downregulated) were identified between the obs6w and deobs10d groups.

In addition, the present study performed DEM and DEG comparisons of the different groups. The Venn plots of DEMs and DEGs of obs10d vs. sham control and obs6w vs. sham control groups are presented in Fig. 1A. DEGs and DEMs with the same expression trends in the obs10d and obs6w groups were considered to be obstruction-associated. The results demonstrated that 664 obstruction-associated genes (420 up and 244 downregulated) exhibited similar expression trends in the obs10d and obs6w groups, compared with sham control. A total of 3 upregulated obstruction-associated miRNAs (rno-miR-34b-star, rno-miR-132 and rno-miR-15b), and 6 downregulated miRNAs (rno-miR-101b, rno-miR-26b, rno-miR-338, rno-miR-338-star, rno-miR-383 and rno-miR-708), were identified and the mean expression value of each is presented in Fig. 1B. The heat maps of the 9 DEMs and 664 DEGs are presented in Fig. 1C and D, respectively.

In addition, the Venn plots of DEMs and DEGs of obs6w vs. sham and obs6w vs. deobs10d groups are presented in Fig. 2A. 
Table II. KEGG pathways enriched by removal of obstruction-associated differentially expressed genes.

A, Upregulated in obs6w vs. sham and downregulated in obs6w vs. deobs10d groups

\begin{tabular}{|c|c|c|c|}
\hline Term & Count & P-value & Genes \\
\hline rno04512: ECM-receptor interaction & 12 & 4.34E-08 & $\begin{array}{l}\text { Thbs4, Itga7, Itga5, Itga8, Col4a1, Col4a2_p1, } \\
\text { Itga4, Lamc1, Sdc3, Hmmr, Lama5, Fn1 }\end{array}$ \\
\hline rno04510: Focal adhesion & 16 & $3.16 \mathrm{E}-07$ & $\begin{array}{l}\text { Zyx, Actn1, Itga7, Col4a1, Col4a2_p1, Lamc1, } \\
\text { Lama5, Flnc, Thbs4, Actn2, Itga5, Ilk, Itga8, } \\
\text { Itga4, Figf, Fn1 }\end{array}$ \\
\hline $\begin{array}{l}\text { rno05412: Arrhythmogenic right ventricular } \\
\text { cardiomyopathy }\end{array}$ & 10 & $2.07 \mathrm{E}-06$ & $\begin{array}{l}\text { Actn2, Actn1, Itga7, Gja1, Itga5, Itga8, } \\
\text { Cacna2d1_p1, Itga4, Cacng7, Sgcg }\end{array}$ \\
\hline rno05414: Dilated cardiomyopathy & 10 & $1.07 \mathrm{E}-05$ & $\begin{array}{l}\text { Itga7, Itga5, Itga8, Cacna2d1_p1, Adcy7, Itga4, } \\
\text { Tgfb2, Cacng7, Sgcg, Tgfb3 }\end{array}$ \\
\hline rno05410: Hypertrophic cardiomyopathy & 9 & $4.88 \mathrm{E}-05$ & $\begin{array}{l}\text { Itga7, Itga5, Itga8, Cacna2d1_p1, Itga4, Tgfb2, } \\
\text { Cacng7, Sgcg, Tgfb3 }\end{array}$ \\
\hline rno04110: Cell cycle & 10 & $1.57 \mathrm{E}-04$ & $\begin{array}{l}\text { Ccne1, Ccnb1, Gadd45g, Cdk1, Ccnb2, Bub1, } \\
\text { Tgfb2, Cdc20, Tgfb3, Ccna2 }\end{array}$ \\
\hline rno04810: Regulation of actin cytoskeleton & 12 & $4.31 \mathrm{E}-04$ & $\begin{array}{l}\text { Actn2, Iqgap3, Actn1, Itga7, Msn, Itga5, Itga8, } \\
\text { Diaph3, Itga4, Rras2_p1, Enah, Fn1 }\end{array}$ \\
\hline rno04010: MAPK signaling pathway & 13 & $9.39 \mathrm{E}-04$ & $\begin{array}{l}\text { Gadd45g, Bdnf, Flnc, Nr4a1, Cacna2d1_p1, } \\
\text { Dusp10, Dusp3, Rras2_p1, Tgfb2, Cacng7, } \\
\text { Dusp1, Tgfb3, Dusp8 }\end{array}$ \\
\hline rno05222: Small cell lung cancer & 7 & $1.94 \mathrm{E}-03$ & $\begin{array}{l}\text { Ccne1, Col4a1, Col4a2_p1, Ptgs2, Lamc1, } \\
\text { Lama5, Fn1 }\end{array}$ \\
\hline rno04115: p53 signaling pathway & 6 & $3.81 \mathrm{E}-03$ & Ccne1, Ccnb1, Gadd45g, Cdk1, Serpine1, Ccnb2 \\
\hline rno04114: Oocyte meiosis & 7 & $8.18 \mathrm{E}-03$ & $\begin{array}{l}\text { Ccne1, Ccnb1, Cdk1, Ccnb2, Bub1, Adcy7, } \\
\text { Cdc20 }\end{array}$ \\
\hline rno05200: Pathways in cancer & 12 & $1.13 \mathrm{E}-02$ & $\begin{array}{l}\text { Ccne1, Arnt2, Col4a1, Col4a2_p1, Slc2a1, Ptgs2, } \\
\text { Lamc1, Tgfb2, Lama5, Tgfb3, Fn1, Figf }\end{array}$ \\
\hline $\begin{array}{l}\text { rno04914: Progesterone-mediated oocyte } \\
\text { maturation }\end{array}$ & 6 & $1.21 \mathrm{E}-02$ & Ccnb1, Cdk1, Ccnb2, Bub1, Adcy7, Ccna2 \\
\hline rno05211: Renal cell carcinoma & 5 & 2.37E-02 & Arnt2, Slc2a1, Tgfb2, Tgfb3, Figf \\
\hline
\end{tabular}

B, Downregulated in obs6w vs. sham and upregulated in obs6w vs. deobs10d groups

\begin{tabular}{lccc}
\hline Term & Count & P-value & Genes \\
\hline $\begin{array}{l}\text { rno04960: Aldosterone-regulated sodium } \\
\text { reabsorption }\end{array}$ & 3 & 0.015502 & Atp1b2, Nr3c2, Scnn1g \\
\hline
\end{tabular}

KEGG, Kyoto Encyclopedia of Genes and Genomes.

The results indicated that 301 genes were upregulated in obs6w vs. sham and downregulated in obs6w vs. deobs10d groups, whereas 69 genes were downregulated in obs6w vs. sham and upregulated in obs6w vs. deobs10d groups. Overall, 370 genes exhibited the opposite expression trend prior to and following the removal of the obstruction. The heat map of these DEGs is presented in Fig. 2B. However, no miRNAs demonstrated opposite expression trends in the obs6w vs. sham and obs6w vs. deobs10d groups.

KEGG pathway analysis. The present study then performed KEGG pathway enrichment analysis for 664 obstruction-associated genes using the DAVID online tool. The results revealed that a series of significant KEGG pathways were enriched, which were primarily associated with focal adhesion, ECM-receptor interaction, arrhythmogenic right ventricular cardiomyopathy (ARVC), dilated cardiomyopathy and vascular smooth muscle contraction (Table I).

Furthermore, it was demonstrated that 301 DEGs, which were upregulated in obs6w vs. sham and downregulated in obs6w vs. deobs10d groups, were primarily enriched in KEGG pathways associated with ECM-receptor interaction, focal adhesion and ARVC (Table IIA). However, DEGs that were downregulated in obs6w vs. sham and upregulated in obs6w vs. deobs10d groups were only significantly enriched in aldosterone-regulated sodium reabsorption (Table IIB), in 
A

miRNA

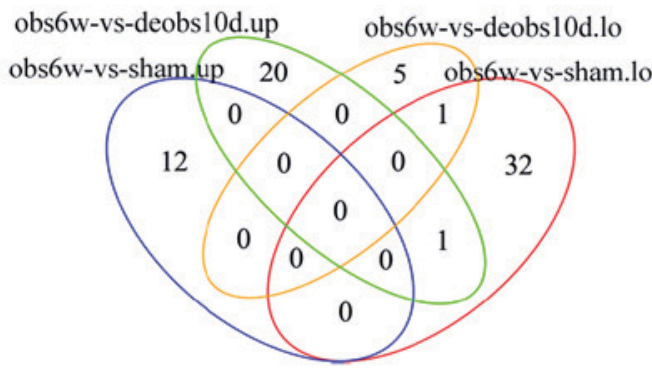

gene

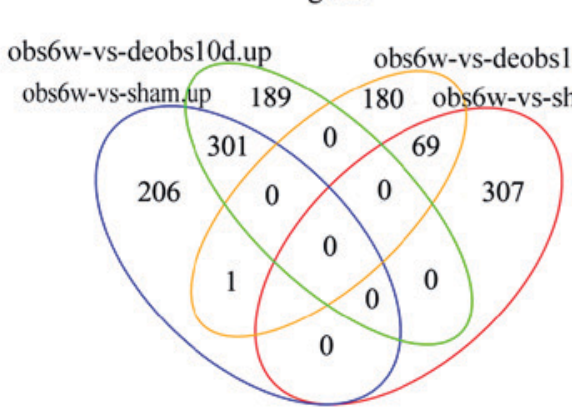

B

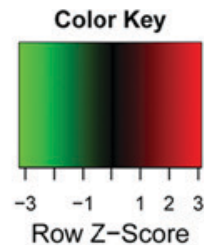

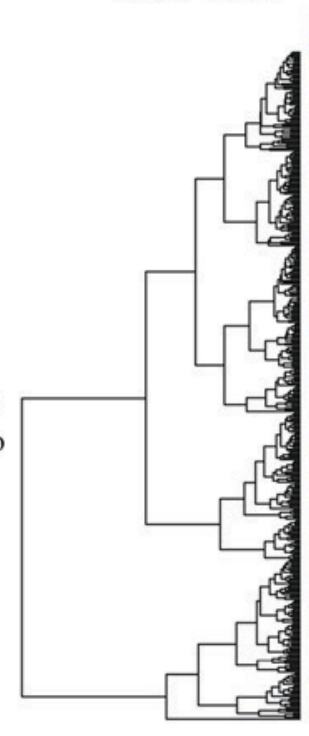

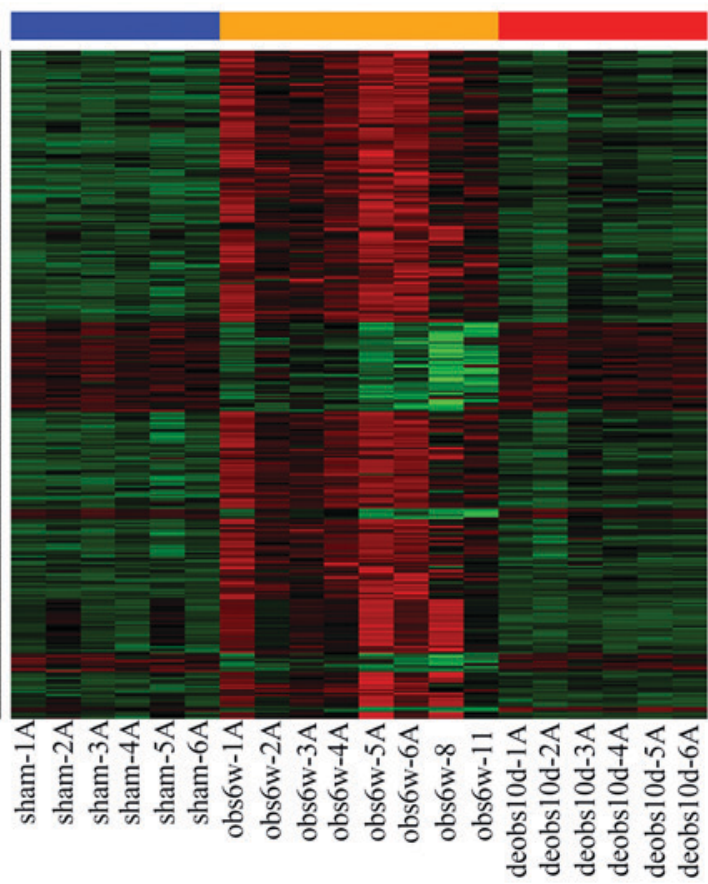

Figure 2. DEMs and DEGs in obs6w group compared with the sham and deobs10d group. (A) Venn plots of DEMs and DEGs. (B) The heat map of DEGs Columns represent rat bladder samples, from the sham10d (blue; $n=6$ ), obs6w (orange; $n=8$ ) and deobs10d (red; $n=6$ ) groups. The colors depict high (red) and low (green) relative level of gene expression. The color alteration from green to black and to red represents increasing expression of DEGs. Right vertical axis represents the clustering of genes. DEGs, differentially expressed genes; DEMs, differentially expressed microRNAs; obs6w, urinary bladder removed 6 weeks following obstruction; deobs10d, obstruction removed 6 weeks following surgery; miRNA, microRNA.

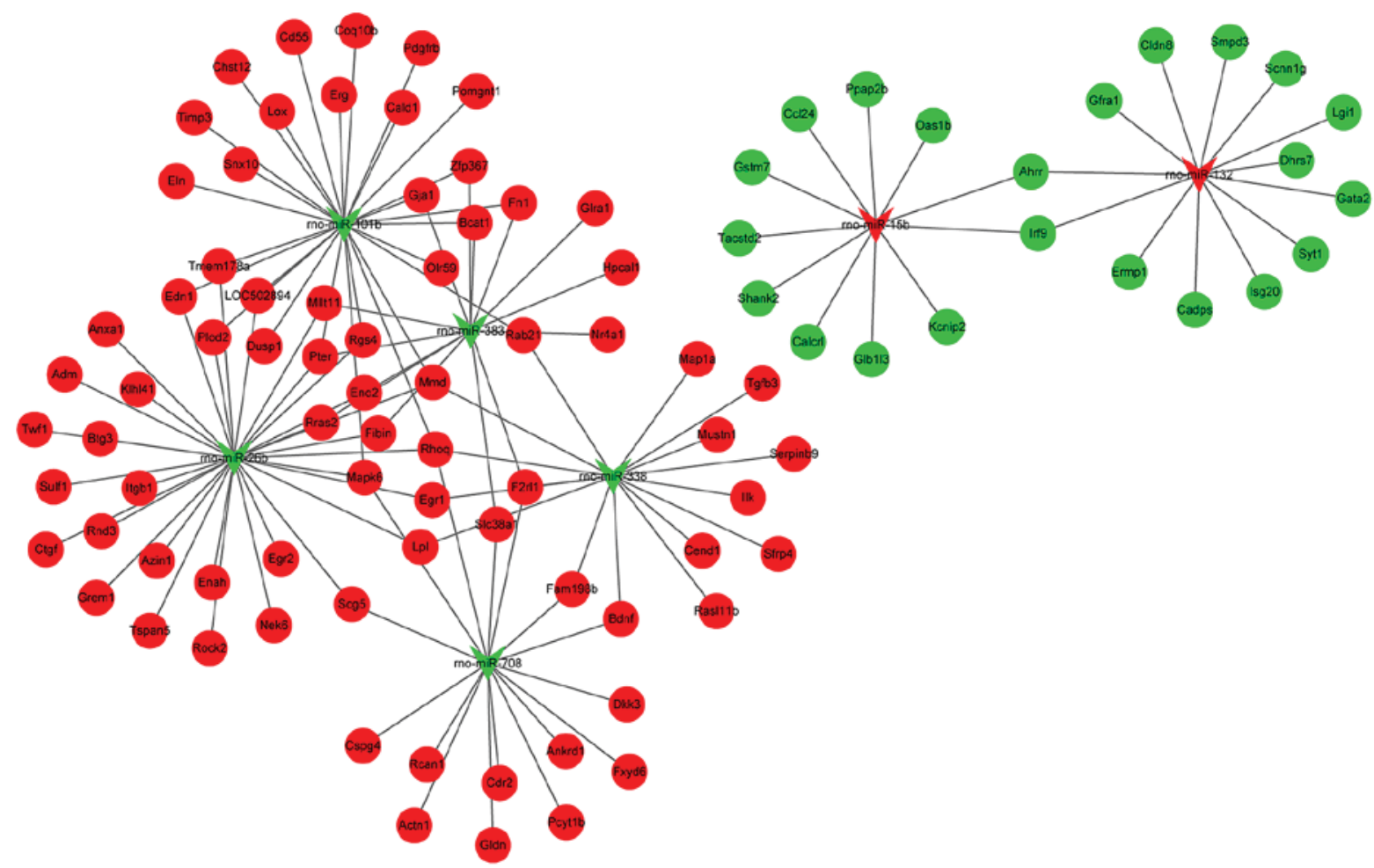

Figure 3. Obstruction-associated DEMs-DEGs regulatory network. Circular nodes, genes; V-shaped nodes, miRs; red, upregulated DEMs or DEGs; green, downregulated DEMs or DEGs. DEGs, differentially expressed genes; DEMs, differentially expressed microRNAs; miR, microRNA. 


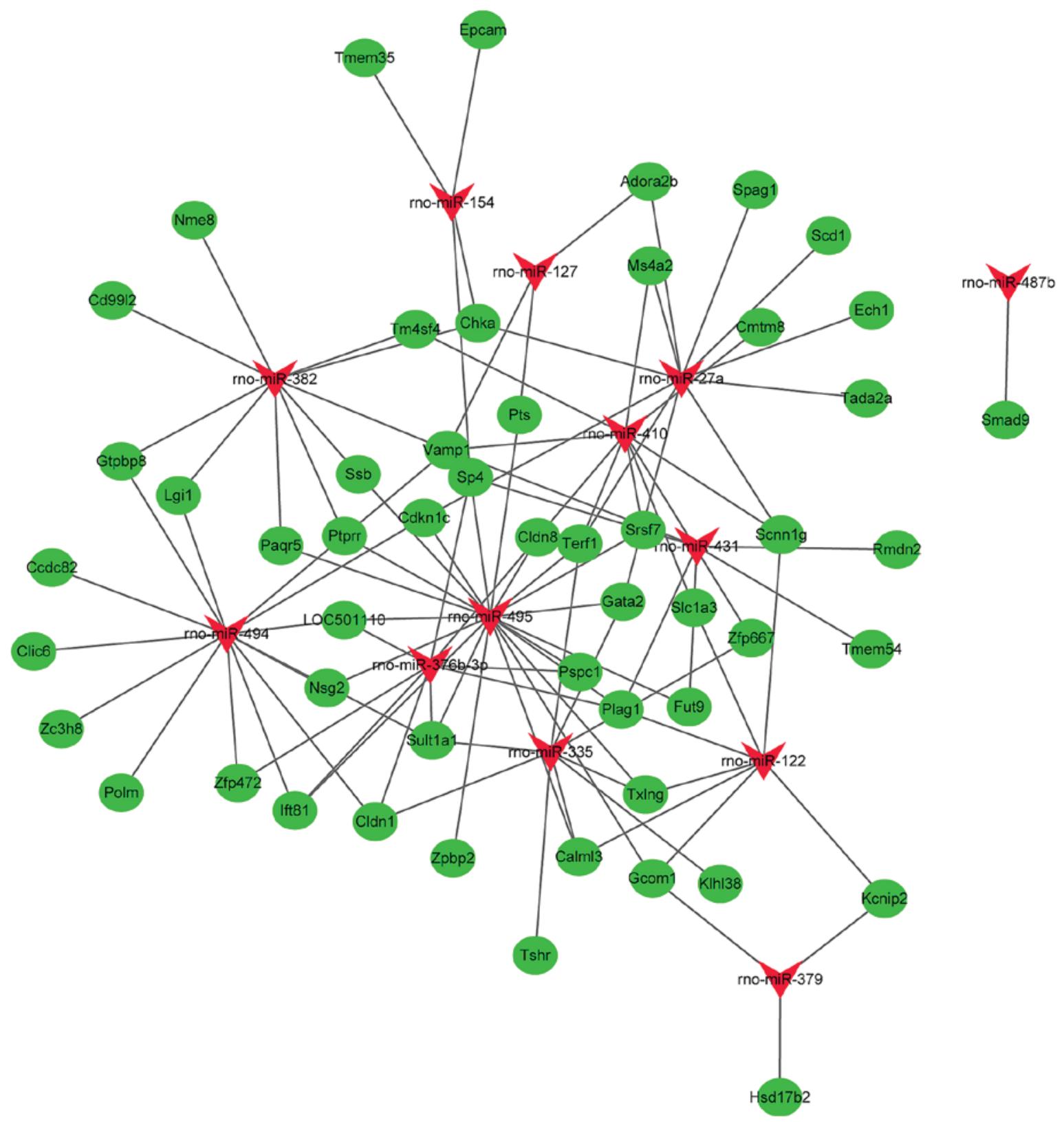

Figure 4. Removal of obstruction-associated DEMs-DEGs regulatory network Circular nodes, genes; V-shaped nodes, miRs; red, upregulated DEMs or DEGs; green, downregulated DEMs or DEGs. DEGs, differentially expressed genes; DEMs, differentially expressed microRNAs; miR, microRNA.

which 3 of 69 DEGs were enriched $\left(\right.$ ATPase $\mathrm{Na}^{+} / \mathrm{K}^{+}$transporting subunit beta 2 , nuclear receptor subfamily 3 group $\mathrm{C}$ member 2, sodium channel epithelial 1 gamma subunit).

Analysis of miRNA-gene interaction network. The present study used miRanda to predict the target genes of miRNAs. Following this, an miRNA-gene interaction network was constructed by integrating the target genes with DEGs. As presented in Fig. 3, the obstruction-associated DEMs-DEGs interaction network was constructed, including 103 nodes, 7 of which were DEMs, and 129 connections. rno-miR-26b and rno-miR-101b were the two largest nodes of the 7 DEMs and interacted with 32 and 27 DEGs, respectively. In addition, the interaction network of obs6w vs. deobs10d group was constructed, including 66 nodes (13 DEMs and 53 DEGs) and 108 connections (Fig. 4). rno-miR-495 and rno-miR-494 were the two largest nodes of the 13 DEMs and targeted 21 and 14 DEGs, respectively. The downregulated gene vesicle-associated membrane protein 1 was regulated by 5 upregulated miRNAs.

\section{Discussion}

The urinary bladder is exposed to mechanical stress from the bladder wall, which results in modification of gene and protein expression profiles in epithelial and smooth muscle cells, altering the ultrastructure and physiology of cells in the bladder wall. Long-term partial BOO alters caldesmon isoform expression levels, which are associated with bladder contractile function (23). The role of prostatic obstruction is important in the development of lower urinary tract symptoms (LUTS), in addition to alterations in bladder detrusor muscle 
physiology. However, the molecular mechanisms underlying this complex process fully remain to be elucidated.

The bladder remodeling process is typically considered to be initiated following $\mathrm{BOO}$ and is important in the development of clinical LUTS. It has previously been demonstrated that during BOO, ECM deposition results in a thickened bladder wall. BOO initiates a physiopathological cascade of events, ultimately leading to urinary tract symptoms. The obstructed bladder modifies its structure to compensate for the increased resistance to flow, and significant hypoxia ensues due to the high resistance to flow and consequent high intravesical pressure, resulting in fibrosis. Further investigations are required to fully understand the reparative process of bladder remodeling for the development of appropriate preventative or remedial treatment.

The present study demonstrated that numerous miRNAs were significantly differentially expressed in the rat urinary bladder following BOO. Upregulated miRNAs included rno-miR-34b-star, rno-miR-132 and rno-miR-15b, and downregulated miRNAs included rno-miR-101b, rno-miR-26b, rno-miR-338, rno-miR-338-star, rno-miR-383 and rno-miR-708. miRNAs have been reported to regulate numerous genes and therefore may be involved in tumorigenesis. miRNA profiling has previously been reported in tumor classification, diagnostics and therapeutics. miR-26b profiling has specifically been reported in angiogenesis in breast cancer cells (24) and epithelial-mesenchymal transition in hepatocellular carcinoma (25). Additionally, miR-26b is downregulated in carcinoma-associated fibroblasts from estrogen receptor-positive breast cancers leading to enhanced cell migration and invasion (26). A further miRNA, miR-101, is downregulated in bladder transitional cell carcinoma and associated with poor prognosis (27), and has been demonstrated to suppress liver fibrosis by targeting the TGF- $\beta$ signaling pathway (28). Another specific miRNA, miR-132, has been revealed to regulate liver fibrosis via an epigenetic relay pathway (29), and serve as a master signaling switch in cardiac fibroblasts (30). BOO increases miR-132/212 expression levels via the transcription factors aryl hydrocarbon receptor and cAMP responsive element binding protein, which are associated with methyl-CpG binding protein 2 repression and cell viability (14). The mRNA microarray analysis performed by Ekman et al (13) indicated that reduction of miR-29 following BOO was associated with increased levels of miR-29 target mRNAs, including mRNAs for tropoelastin, the matricellular proteins secreted protein acidic and rich in cysteine and collagen IV.

Various miRNAs have been characterized for their important roles in focal adhesion, ECM-receptor interaction, cell adhesion molecules and regulation of the actin cytoskeleton. miR-29 repression has been reported to be involved in the pathogenesis of ECM remodeling and altered stiffness (13). To obtain insights into the classification of miRNAs, the present study conducted GO analysis to predict target genes. The results revealed various GO terms associated with signal transduction of focal adhesion, ECM-receptor interaction, cell adhesion molecules and regulation of the actin cytoskeleton. In addition, it was observed that various signal transduction pathways were involved in numerous types of cancer, including the 553 , mitogen activated protein kinase and TGF- $\beta$ signaling pathways. The TGF- $\beta$ signaling pathway may be responsible for the pathological alterations observed in the bladder remodeling and may provide a strategy for therapeutic intervention, following further investigation. The TGF- $\beta$ signaling pathway, which has been reported to be associated with organ fibrosis (31), was additionally observed in the present study, as shown in Table I. It has previously been demonstrated that BOO activates TGF- $\beta$ signaling, leading to mothers against decapentaplegic 2/3 and extracellular-signal-regulated kinase phosphorylation and increases mRNA levels of established TGF- $\beta$ targets, including connective tissue growth factor (13). Furthermore, outlet obstruction increases TGF- $\beta$ mRNA levels (32) and studies on genetically modified mice have demonstrated a role of TGF- $\beta$ receptor II in the stiffness alteration that follows chronic obstruction (33). A series of genes associated with urinary bladder remodeling have been identified with the development of modern technology in this field.

In conclusion, the present study revealed the dysregulation of miRNA and mRNA expression levels in the urinary bladder following BOO. Bioinformatics-based analysis of these alterations may provide a useful tool for understanding the molecular mechanisms underlying bladder dysfunction. The dysfunctional interactions between DEMs-DEGs may result in the occurrence and progression of this condition. However, further investigations are required to verify the exact roles of the identified miRNA and genes in the pathogenesis of bladder remodeling.

\section{Acknowledgements}

The present study was supported by the Foundation of Shanghai Science and Technology Committee (grant nos. 134119a0600 and 14430720800) and the National Natural Science Foundation of China (grant no. 81570684).

\section{Referen\ces}

1. Boyle P, Robertson C, Mazzetta C, Keech M, Hobbs FD, Fourcade R, Kiemeney L and Lee C; UrEpik Study Group: The prevalence of lower urinary tract symptoms in men and women in four centres. The UrEpik study. BJU Int 92: 409-414, 2003.

2. Zeng J, Pan C, Jiang C and Lindström S: Cause of residual urine in bladder outlet obstruction: An experimental study in the rat. J Urol 188: 1027-1032, 2012.

3. Collado A, Batista E, Gelabert-Más A, Corominas JM, Arañó P and Villavicencio $\mathrm{H}$ : Detrusor quantitative morphometry in obstructed males and controls. J Urol 176: 2722-2728, 2006.

4. Mirone V, Imbimbo C, Longo $\mathrm{N}$ and Fusco F: The detrusor muscle: An innocent victim of bladder outlet obstruction. Eur Urol 51: 57-66, 2007.

5. Valencia-Sanchez MA, Liu J, Hannon GJ and Parker R: Control of translation and mRNA degradation by miRNAs and siRNAs. Genes Dev 20: 515-524, 2006.

6. Catto JW, Alcaraz A, Bjartell AS, De Vere White R, Evans CP Fussel S, Hamdy FC, Kallioniemi O, Mengual L, Schlomm T and Visakorpi T: MicroRNA in prostate, bladder, and kidney cancer: A systematic review. Eur Urol 59: 671-681, 2011.

7. Alvarez-Garcia I and Miska EA: MicroRNA functions in animal development and human disease. Development 132: 4653-4662, 2005.

8. Persson L: Radiation risks are evaluated. There is no totally harmless level. Lakartidningen 87: 1264-1266, 1990 (In Swedish).

9. Chen JF, Mandel EM, Thomson JM, Wu Q, Callis TE, Hammond SM, Conlon FL and Wang DZ: The role of microRNA-1 and microRNA-133 in skeletal muscle proliferation and differentiation. Nat Genet 38: 228-233, 2006. 
10. Roderburg C,Luedde M,Vargas Cardenas D, Vucur M,MollnowT, Zimmermann HW, Koch A, Hellerbrand C, Weiskirchen R, Frey $\mathrm{N}$, et al: miR-133a mediates TGF- $\beta$-dependent derepression of collagen synthesis in hepatic stellate cells during liver fibrosis. J Hepatol 58: 736-742, 2013.

11. Bartel DP: MicroRNAs: Genomics, biogenesis, mechanism, and function. Cell 116: 281-297, 2004.

12. Canturk KM, Ozdemir M, Can C, Öner S, Emre R, Aslan H, Cilingir O, Ciftci E, Celayir FM, Aldemir O, et al: Investigation of key miRNAs and target genes in bladder cancer using miRNA profiling and bioinformatic tools. Mol Biol Rep 41: 8127-8135, 2014.

13. Ekman M, Bhattachariya A, Dahan D, Uvelius B, Albinsson S and Swärd K: Mir-29 repression in bladder outlet obstruction contributes to matrix remodeling and altered stiffness. PLoS One 8: e82308, 2013.

14. Sadegh MK, Ekman M, Krawczyk K, Svensson D, Göransson O, Dahan D, Nilsson BO, Albinsson S, Uvelius B and Swärd K: Detrusor induction of miR-132/212 following bladder outlet obstruction: Association with MeCP2 repression and cell viability. PLoS One 10: e0116784, 2015.

15. Duan LJ, Qi J, Kong XJ, Huang T, Qian XQ, Xu D, Liang JH and Kang J: MiR-133 modulates TGF- $\beta 1$-induced bladder smooth muscle cell hypertrophic and fibrotic response: Implication for a role of microRNA in bladder wall remodeling caused by bladder outlet obstruction. Cell Signal 27: 215-227, 2015.

16. Gautier L, Cope L, Bolstad BM and Irizarry RA: Affy-analysis of Affymetrix GeneChip data at the probe level. Bioinformatics 20 : 307-315, 2004

17. Irizarry RA, Hobbs B, Collin F, Beazer-Barclay YD, Antonellis KJ, Scherf U and Speed TP: Exploration, normalization, and summaries of high density oligonucleotide array probe level data. Biostatistics 4: 249-264, 2003.

18. Smyth G: Limma: Linear models for microarray data. In: Bioinformatics and Computational Biology Solutions using $\mathrm{R}$ and Bioconductor. Gentleman R, Carey V, Huber W, Irizarry R, Dudoit, S (eds). Springer: New York, NY, pp97-420, 2005.

19. Benjamini Y and Hochberg Y: Controlling the False discovery rate: A practical and powerful approach to multiple testing. J R Stat Soc. Series B (Methodological) 57: 289-300, 1995.

20. Scales M, Jäger R, Migliorini G, Houlston RS and Henrion MY: visPIG-a web tool for producing multi-region, multi-track, multi-scale plots of genetic data. PLoS One 9: e107497, 2014.

21. Kanehisa M, Goto S, Sato Y, Furumichi M and Tanabe M: KEGG for integration and interpretation of large-scale molecular data sets. Nucleic Acids Res 40 (Database Issue): D109-D114, 2012.

22. Huang da W, Sherman BT and Lempicki RA: Systematic and integrative analysis of large gene lists using DAVID bioinformatics resources. Nat Protoc 4: 44-57, 2009.
23. Yang L, He DL, Wang S, Cheng HP and Wang XY: Effect of long-term partial bladder outlet obstruction on caldesmon isoforms and their correlation with contractile function. Acta Pharmacol Sin 29: 600-605, 2008.

24. Anbalagan D, Yap G, Yuan Y, Pandey VK, Lau WH, Arora S, Bist P, Wong JS, Sethi G, Nissom PM, et al: Annexin-A1 regulates microRNA-26b* and microRNA-562 to directly target $\mathrm{NF}-\kappa \mathrm{B}$ and angiogenesis in breast cancer cells. PLoS One 9: e114507, 2014

25. Shen G, Lin Y, Yang X, Zhang J, Xu Z and Jia H: MicroRNA-26b inhibits epithelial-mesenchymal transition in hepatocellular carcinoma by targeting USP9X. BMC Cancer 14: 393, 2014.

26. Verghese ET, Drury R, Green CA, Holliday DL, Lu X, Nash C, Speirs V, Thorne JL, Thygesen HH, Zougman A, et al: MiR-26b is down-regulated in carcinoma-associated fibroblasts from ER-positive breast cancers leading to enhanced cell migration and invasion. J Pathol 231: 388-399, 2013.

27. Zhang H, Qi F, Cao Y, Chen M and Zu X: Down-regulated microRNA-101 in bladder transitional cell carcinoma is associated with poor prognosis. Med Sci Monit 20: 812-817, 2014

28. Tu X, Zhang H, Zhang J, Zhao S, Zheng X, Zhang Z, Zhu J, Chen J, Dong L, Zang Y and Zhang J: MicroRNA-101 suppresses liver fibrosis by targeting the TGF $\beta$ signalling pathway. J Pathol 234: 46-59, 2014

29. Mann J, Chu DC, Maxwell A, Oakley F, Zhu NL, Tsukamoto H and Mann DA: MeCP2 controls an epigenetic pathway that promotes myofibroblast transdifferentiation and fibrosis. Gastroenterology 138: 705-714, 714.e1-4, 2010.

30. Eskildsen TV, Schneider M, Sandberg MB, Skov V, Brønnum H, Thomassen M, Kruse TA, Andersen DC and Sheikh SP: The microRNA-132/212 family fine-tunes multiple targets in Angiotensin II signalling in cardiac fibroblasts. J Renin Angiotensin Aldosterone Syst 16: 1288-1297, 2015.

31. Wang K, Tang Y, Yan F, Zhu J and Li J: Potent inhibition of TGF- $\beta$ signaling pathway regulator Abl: Potential therapeutics for hepatic fibrosis. J Recept Signal Transduct Res 35: 410-419, 2015.

32. Baskin LS, Sutherland RS, Thomson AA, Hayward SW and Cunha GR: Growth factors and receptors in bladder development and obstruction. Lab Invest 75: 157-166, 1996.

33. Anumanthan G, Tanaka ST, Adams CM, Thomas JC, Wills ML, Adams MC, Hayward SW, Matusik RJ, Bhowmick NA, Brock JW III and Pope JC IV: Bladder stromal loss of transforming growth factor receptor II decreases fibrosis after bladder obstruction. J Urol 182 (4 Suppl): S1775-S1780, 2009. 